
Brasileira 24: 481-484.

\title{
Qualidade dos frutos de tomate de mesa quando submetidos a impacto por ocasião do beneficiamento
}

\author{
Ana Maria de Magalhães; Marcos David Ferreira \\ Unicamp-FEA, C. Postal 6011, 13083-875 Campinas-SP; E-mail: anamaria@agr.unicamp.br
}

\section{RESUMO}

A tomaticultura de mesa vem se modernizando nos últimos anos, com a implantação de unidades de beneficiamento e classificação que limpam, selecionam e classificam os produtos. Apesar destas mudanças, as perdas pós-colheitas ainda são altas, principalmente pela incidência de danos físicos, que ocorrem em pontos de transferência nos equipamentos e durante o processo de embalagem. Este trabalho teve como objetivo avaliar a qualidade dos frutos de tomate de mesa, cv "Débora", quando submetidos à queda após a etapa de limpeza em protótipo desenvolvido em laboratório para simular este sistema, relacionando alterações na qualidade com a magnitude de impacto encontrada $(\mathrm{G}, \mathrm{m} / \mathrm{s} 2)$. Para tanto tomates foram beneficiados em rotações de $100 \mathrm{rpm}$ e submetidos à queda em três diferentes alturas $(10,20$ e $30 \mathrm{~cm})$ e três diferentes superfícies receptoras (espuma, caixa plástica e metálica). A magnitude dos impactos (G, $\mathrm{m} / \mathrm{s} 2$ ) foi mensurada através da utilização da esfera instrumentada de 70 mm (Techmark, Inc., Lansing, EUA). A qualidade dos frutos após serem submetidos ao impacto foi avaliada por meio da perda de massa (\%), injúria interna e de análises químicas. Os resultados obtidos com a esfera instrumentada mostraram que a superfície de espuma reduziu a aceleração máxima $(\mathrm{G}, \mathrm{m} / \mathrm{s} 2)$ em aproximadamente $70 \%$, para as alturas de queda de 10 e $20 \mathrm{~cm}$ e de $47 \%$ para a queda de $30 \mathrm{~cm}$, quando comparada à superfície metálica. Nos ensaios com os tomates, observou-se que frutos submetidos à queda de $30 \mathrm{~cm}$ em superfície metálica apresentaram um aumento de $31 \%$ nos danos internos quando comparados aos frutos submetidos à queda desta mesma altura em superfície de espuma. A perda de massa foi maior $(3,46 \%)$ nos frutos submetidos à queda de $30 \mathrm{~cm}$ na superfície caixa plástica. Para as análises químicas não foi observada diferença estatística entre as três superfícies receptoras e as três alturas de queda. Por meio dos resultados obtidos, conclui-se que o uso de superfícies protetoras diminui significativamente os danos internos nos frutos e que tomates submetidos à queda em caixas plásticas demonstraram significativa incidência em danos físicos, portanto, modificações devem ser realizadas na etapa de embalagem.

Palavras-chave: Lycopersicon esculentum, galpões de beneficiamento, danos físicos.

\section{ABSTRACT}

Fresh market tomatoes quality when submitted to impact

The cultivation of fresh market tomatoes has been modernized with the introduction of packing lines for cleaning, sorting and classifying. Despite of those technological changes, post harvest losses are still high, mainly due to the incidence of the mechanical damages that occurr in the transfer points and during packing. The main goal of this research was to evaluate tomato quality of cv. Debora, subject to dropping after being subjected to the cleaning step on a prototype developed in laboratory to simulate a commercial system, relating quality changes to impact magnitude $(\mathrm{G}, \mathrm{m} / \mathrm{s} 2)$. Therefore, fruits were subject to a $100 \mathrm{rpm}$ rotation and submitted to a fall from three different heights $(10,20$ e $30 \mathrm{~cm}$ ) and three different surfaces (foam, plastic box and metallic sheet). The impact magnitude $(\mathrm{G}, \mathrm{m} / \mathrm{s} 2)$ was measured using an instrumented sphere, $70 \mathrm{~mm}$ Techmark, Inc., Lansing, EUA). Fruit quality after impact was evaluated by weight loss (\%), internal bruising, and chemical analyses. The use of an instrumented sphere showed that a foam surface decreases maximum acceleration $(\mathrm{G}, \mathrm{m} /$ s2) by about $70 \%$ for 10 and $20 \mathrm{~cm}$ high drops and $47 \%$ for $30 \mathrm{~cm}$ drop, compared to a metallic surface. Tomatoes submitted to drops from $30 \mathrm{~cm}$ high over metal surface showed increase on $31 \%$ on internal bruising when compared to fruits submitted to drops at the same height on the covered surface (foam). The highest weight loss $(3,46 \%)$ was found in fruits submitted to a drop from $30 \mathrm{~cm}$ on plastic boxes. No statistical differences were observed between the different treatments. Based on the results, it could be concluded that the use of protective surfaces significantly decrease internal bruising and weight loss and tomatoes submitted to drops in plastic boxes showed a significant increase in physical damage, therefore, changes have to be made at the packaging stage.

Keywords: Lycopersicon esculentum, packing line, mechanical damages.

\section{(Recebido para publicação em 13 de janeiro de 2006; aceito em 31 de outubro de 2006)}

$\mathrm{O}$ tomate é um produto de grande importância econômica, sendo que a sua produção mundial em 2004 foi de aproximadamente 116 milhões de toneladas. O Brasil ficou em oitavo lugar com uma produção de 3.394.677 toneladas, cultivada em aproximadamente 57.500 ha (FAO, 2005). Apesar das mudanças tecnológicas ocorridas durante o processo de beneficiamento e clas- sificação para o tomate de mesa, as perdas pós-colheita ainda continuam altas. Uma das principais razões para isto relaciona-se à incidência de danos físicos que ocorrem quando os frutos são submetidos a quedas em superfícies protegidas ou não e com outros frutos. A severidade dos danos causados por impactos em uma linha de classificação pode ser reduzida diminuindo a altura de ele- vação de queda entre as etapas ou através da utilização de protetores, os quais podem dissipar a força de impacto (Hyde \& Zhang, 1992). Os impactos em uma linha de classificação podem ser mensurados através da esfera instrumentada, que é um equipamento plástico dotado de um registrador de aceleração. Os níveis de impactos são medidos na aceleração máxima $(\mathrm{G}=$ 


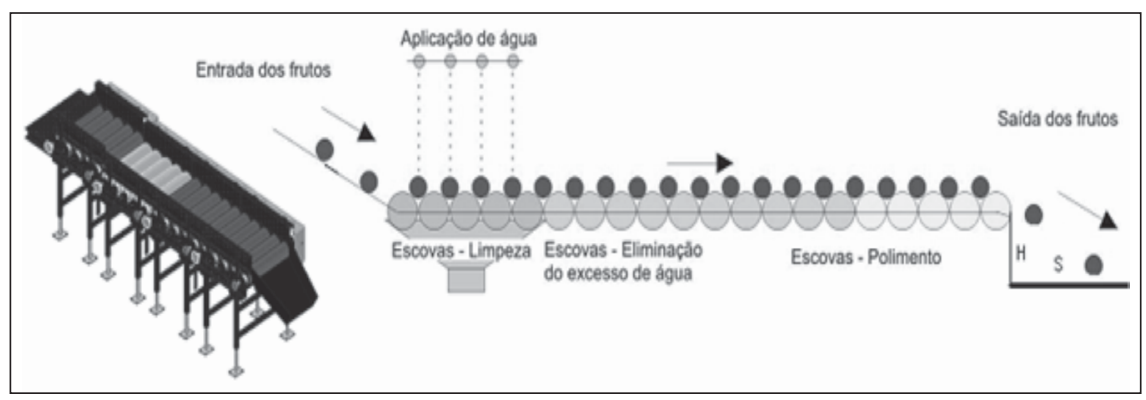

Figura 1. Protótipo desenvolvido para o sistema UNIMAC. $\mathrm{H}=$ altura de queda; s= superfície de queda.

$9,81 \mathrm{~m} / \mathrm{s} 2)$ e mudanças na velocidade (Av) $(\mathrm{m} / \mathrm{s})$. O Av pode ser um indicativo da superfície de impacto, por exemplo, quanto mais baixa a velocidade mais rígida e dura é a superfície de impacto. Todavia, velocidade é também positivamente correlacionada com a aceleração máxima: à medida que se aumenta a altura de queda maior será a aceleração máxima e a velocidade (Sargent et al., 1992). Nos frutos, os danos físicos causam deformações plásticas, rupturas superficiais chegando até a destruição dos tecidos vegetais. Além dos danos diretos, a incidência de ferimentos em frutas e hortaliças pode levar a um aumento de doenças pós-colheita e alterações fisiológicas e químicas (Honório $\&$ Moretti, 2002). Em tomates a incidência e a severidade de danos são cumulativas nas práticas de manuseio póscolheita. A ocorrência destes danos pode causar o aparecimento de desordem fisiológica que alteram o curso normal de amadurecimento (Halsey, 1955). Os frutos de tomate com esta desordem apresentam o tecido locular em colapso, desorganizado, com coloração verde amarelada, de aspecto turvo e, em casos mais severos, grande perda de água (Hatton \& Reeder, 1963). Vários trabalhos têm demonstrado que tomates com desordem fisiológica causada por impacto apresentam alterações significativas em acidez titulável, vitamina $\mathrm{C}$ total, atividade enzimática, carotenóides totais e firmeza (Moretti et al., 1998). Este trabalho teve o objetivo de mensurar a magnitude de impacto $(\mathrm{G}, \mathrm{m} / \mathrm{s} 2)$ e avaliar a incidência de danos físicos e alterações na qualidade dos frutos após serem submetidos a diferentes alturas de queda e superfícies receptoras na etapa de limpeza, em protótipo desenvolvido para simular esta fase em laboratório.

\section{MATERIAL E MÉTODOS}

Os ensaios foram realizados no protótipo desenvolvido na Faculdade de Engenharia Agrícola/UNICAMP para o sistema UNIMAC-Unidade Móvel de Auxílio à Colheita, na etapa de limpeza (Figura 1). O equipamento dispõe de grupos de escovas com diferentes funções, como está esquematizado na Figura 1 . O primeiro grupo é formado por escovas sintéticas que, associados com o sistema de aspersão de água, tem a função de remover as impurezas superficiais dos frutos. O segundo grupo dispõe de escovas específicas para a eliminação do excesso de água. Em seguida vêm dois grupos formados por escovas de pêlo de animal, responsáveis pelo polimento.

Em uma primeira etapa para medição da magnitude de impacto, realizouse ensaio utilizando a esfera instrumentada de diâmetro $70 \mathrm{~mm}$ (Techmark, Inc., Lansing, EUA), que consiste em um equipamento plástico com registrador de aceleração para a avaliação da magnitude de impactos $(\mathrm{G}$, $\mathrm{m} / \mathrm{s} 2$ ). A esfera foi colocada juntamente com tomates com semelhante diâmetro na rotação de $100 \mathrm{rpm}$, e submetida à queda em três diferentes alturas (10, 20 e $30 \mathrm{~cm}$ ) e em três superfícies receptoras, (espuma: espuma coberta com courvim $8 \mathrm{~mm}$, caixa plástica $(52 \times 32 \times 28 \mathrm{~cm})$ : utilizada comercialmente no transporte de tomate de mesa, metálica: aço carbono $5 \mathrm{~mm}$ ). A rotação de 100 rpm é a recomendada para o beneficiamento de frutas e hortaliças (Miller et al., 2001). O tempo de permanência da esfera no protótipo foi monitorado com um cronômetro de pre- cisão e cada ensaio de queda repetido cinco vezes. Após o ensaio, os dados foram transferidos para 0 microcomputador e através dos dados médios da aceleração máxima determinou-se a magnitude do impacto. Os dados coletados para a esfera instrumentada foram apresentados na forma de gráficos, relacionando a aceleração média $(\mathrm{G}, \mathrm{m} / \mathrm{s} 2)$ aos diferentes tratamentos.

$\mathrm{Na}$ segunda etapa, o ensaio foi realizado utilizando tomates de mesa, cultivar Débora, colhidos diretamente da planta no estádio de maturação salada (CEAGESP, 2000), e transportados cuidadosamente até o laboratório da Faculdade de Engenharia Agrícola/ UNICAMP, evitando que os frutos sofressem danos físicos durante o transporte. O ensaio foi realizado utilizando-se os mesmos tratamentos aplicados com as esferas.

Após 08 dias de armazenamento em temperatura ambiente $\left(23^{\circ} \mathrm{C}\right)$ para avaliar a qualidade dos frutos submetidos a impacto, foi realizada a avaliação da injúria interna de impacto, análises químicas (acidez titulável, $\mathrm{pH}$, sólidos solúveis e vitamina $\mathrm{C}$ ) e perda de massa (\%). A avaliação da injúria interna de impacto "Internal Bruising" foi realizada segundo a metodologia descrita por Sargent et al. (1992). Cada tomate foi cortado na altura do equador e avaliado usando a seguinte escala: $1=$ nenhum dano físico; 2= mínima descoloração do tecido locular; $3=$ observada descoloração do pericarpo e deformação do tecido locular; 4= tecido locular separado dos demais. A perda de massa (\%) foi avaliada utilizando a relação entre a diferença entre massa inicial e final. Para as análises químicas acidez titúlavel total, pH, concentração de sólidos solúveis totais foram utilizados os métodos descritos por Carvalho et al. (1990). Para a análise de vitamina $\mathrm{C}$, a determinação foi feita através de titulação com 2,6-Diclorofenol-Indofenol (Benassi \& Antunes, 1988).

Os ensaios foram planejados a partir de Delineamento Inteiramente Casualizado (DIC) em esquema fatorial. Os fatores considerados nos ensaios foram três alturas de queda e três superfícies receptoras, com cinco repetições 
para a esfera e vinte repetições para o tomate de mesa. Os resultados foram avaliados com análise de variância e encontrando diferença significativa foi aplicado como teste de média, o Tukey a $5 \%$ de probabilidade.

\section{RESULTADOS E DISCUSSÃO}

Nos ensaios com a esfera instrumentada, a superfície metálica apresentou os maiores valores de aceleração máxima $(\mathrm{G}, \mathrm{m} / \mathrm{s} 2)$, seguidos da superfície caixa plástica e por fim da superfície de espuma para as três alturas de queda (Figura 2; $<<0,05$ ). Sargent et al. (1992), também observaram maior valor de aceleração máxima para quedas em superfícies metálicas.

A redução apresentada na aceleração $(\mathrm{G}, \mathrm{m} / \mathrm{s} 2)$ pela superfície de espuma foi de aproximadamente $70 \%$ para as alturas de queda de 10 e $20 \mathrm{~cm}$ e $47 \%$ para a altura de $30 \mathrm{~cm}$ quando comparada à superfície metálica. Timm \& Brown (1991) também relatam uma diminuição no impacto em linhas de classificação de abacate, mamão e abacaxi, quando da utilização de protetores de superfície.

Os tomates que foram submetidos à queda de $30 \mathrm{~cm}$ na superfície de espuma apresentaram uma redução de $26 \%$ nos danos internos quando comparadas às outras duas superfícies (caixa plástica e metálica). Para a queda de $20 \mathrm{~cm}$, a superfície de espuma apresentou uma redução de $13 \%$ com relação à superfície metálica e $6 \%$ com relação a caixa plástica (Tabela 1). Portanto, frutos submetidos à quedas em pontos de transferência em superfícies metálicas e em caixas plásticas estão sujeitos ao aumento significativo da incidência de danos físicos internos. Neste caso, a magnitude de impacto (Figura 2) pode ser relacionada à incidência de danos físicos, pois, com exceção da queda na superfície de espuma, observou-se um aumento crescente, em alguns casos não significativos, da injúria interna, com o aumento da aceleração máxima $(\mathrm{G}, \mathrm{m} /$ s2). Todavia, a superfície protetora mostrou maior influencia na absorção de impactos do que comparativamente na diminuição da incidência em danos físicos internos. Neste ensaio, para a

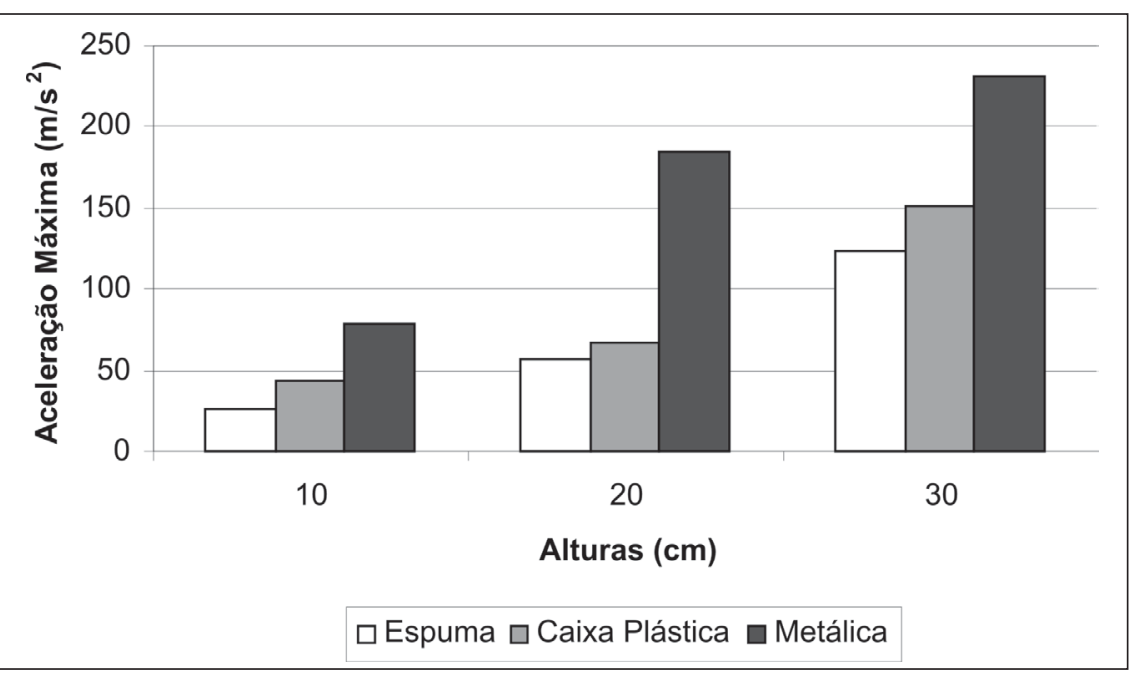

Figura 2. Aceleração máxima encontrada após a queda, utilizando-se a esfera instrumentada, para as três alturas de queda e as três superfícies receptoras.

Tabela 1. Injúria interna e perda de massa (\%), obtidos para os tomates Débora submetidos a diferentes alturas de queda e superfícies, Campinas, 2006.

\begin{tabular}{lcc}
\hline \multirow{2}{*}{ Tratamentos } & \multicolumn{2}{c}{ Análises $^{*}$} \\
\cline { 2 - 3 } & Injúria Interna* & Perda de massa (\%) \\
\hline Testemunha & $1,57 \mathrm{a}$ & $2,82 \mathrm{ab}$ \\
$\mathrm{H}=10 \mathrm{~cm}$ e s= espuma & $2,13 \mathrm{ab}$ & $2,99 \mathrm{ab}$ \\
$\mathrm{H}=20 \mathrm{~cm}$ e $\mathrm{s}=$ espuma & $2,20 \mathrm{ab}$ & $2,97 \mathrm{ab}$ \\
$\mathrm{H}=30 \mathrm{~cm}$ e s= espuma & $2,07 \mathrm{ab}$ & $3,00 \mathrm{ab}$ \\
$\mathrm{H}=10 \mathrm{~cm}$ e s= caixa plástica & $2,13 \mathrm{ab}$ & $2,63 \mathrm{a}$ \\
$\mathrm{H}=20 \mathrm{~cm}$ e s= caixa plástica & $2,33 \mathrm{ab}$ & $2,89 \mathrm{ab}$ \\
$\mathrm{H}=30 \mathrm{~cm}$ e s= caixa plástica & $2,79 \mathrm{~b}$ & $3,46 \mathrm{~b}$ \\
$\mathrm{H}=10 \mathrm{~cm}$ e s= metálica & $2,13 \mathrm{ab}$ & $2,89 \mathrm{ab}$ \\
$\mathrm{H}=20 \mathrm{~cm}$ e s= metálica & $2,53 \mathrm{~b}$ & $3,01 \mathrm{ab}$ \\
$\mathrm{H}=30 \mathrm{~cm}$ e s= metálica & $2,80 \mathrm{~b}$ & $2,84 \mathrm{ab}$ \\
\hline
\end{tabular}

*Médias com mesma letra não diferem entre si pelo teste de Tukey $(\mathrm{p}=0,05) . \mathrm{H}=$ altura de queda; $\mathrm{s}=$ superfície de recepção. ** Média da escala de nota: $1=$ nenhum dano físico; $2=$ mínima descoloração do tecido locular; $3=$ observada descoloração do pericarpo e deformação do tecido locular; $4=$ tecido locular separado dos demais.

maior altura $(30 \mathrm{~cm})$, a redução na magnitude de impacto pela superfície com espuma em relação à metálica apresentou um decréscimo em $47 \%$ e em danos físicos internos de $26 \%$, apresentando uma relação próxima a 2:1, a qual não foi observada nas demais alturas de queda. Nestas observou-se uma relação ainda superior, demonstrando a importância da utilização de superfícies de proteção em linhas de beneficiamento, em especial para maiores alturas. Porém, os dados obtidos indicam, para uma melhor conservação do produto e menores riscos de perdas, que é melhor associar menores alturas com superfícies protetoras. Para Sargent et al. (1992), modi- ficações em alguns pontos da linha de classificação de tomates de mesa, através da utilização de menores pontos de queda e de protetores de superfície, proporcionaram redução em até $50 \%$ na pressão de impacto, com conseqüente diminuição nos danos físicos.

No final do armazenamento, a maior perda de massa $(3,46 \%)$ foi obtida pelos frutos que foram submetidos à queda de $30 \mathrm{~cm}$ na superfície caixa plástica. De um modo geral, a perda de massa dos frutos foi alta $(2,82$ a $3,46 \%)$, não apresentando uma diferença acentuada entre os tratamentos (Tabela 1). A proporção de redução encontrada na magnitude do impacto $(\mathrm{G}, \mathrm{m} / \mathrm{s} 2)$ não foi ob- 
Tabela 2: Acidez titulável, pH, sólidos solúveis e vitamina C, obtidos para os tomates Débora submetidos a diferentes alturas de queda e superfícies, Campinas, 2006.

\begin{tabular}{lcccc}
\hline & \multicolumn{4}{c}{ Análises Químicas* } \\
\cline { 2 - 5 } Tratamentos & $\begin{array}{c}\text { Acidez } \\
\text { titulável } \\
\text { (g ácido } \\
\text { cítrico/100ml) }\end{array}$ & pH & $\begin{array}{c}\text { Sólidos } \\
\text { Solúveis } \\
\text { ('Brix) }\end{array}$ & $\begin{array}{c}\text { Vitamina C } \\
\text { (mg ácido } \\
\text { ascórbico/ } \\
\mathbf{1 0 0 g} \text { ) }\end{array}$ \\
\hline Testemunha & $0,25 \mathrm{a}$ & $4,40 \mathrm{a}$ & $4,72 \mathrm{a}$ & $21,00 \mathrm{a}$ \\
$\mathrm{H}=10 \mathrm{~cm}$ e s= espuma & $0,28 \mathrm{a}$ & $4,45 \mathrm{a}$ & $4,95 \mathrm{ab}$ & $21,05 \mathrm{a}$ \\
$\mathrm{H}=20 \mathrm{~cm}$ e s= espuma & $0,27 \mathrm{a}$ & $4,29 \mathrm{a}$ & $5,00 \mathrm{ab}$ & $21,49 \mathrm{a}$ \\
$\mathrm{H}=30 \mathrm{~cm}$ e s= espuma & $0,28 \mathrm{a}$ & $4,29 \mathrm{a}$ & $4,95 \mathrm{ab}$ & $21,21 \mathrm{a}$ \\
$\mathrm{H}=10 \mathrm{~cm}$ e s= caixa plástica & $0,27 \mathrm{a}$ & $4,33 \mathrm{a}$ & $4,95 \mathrm{ab}$ & $21,04 \mathrm{a}$ \\
$\mathrm{H}=20 \mathrm{~cm}$ e s= caixa plástica & $0,28 \mathrm{a}$ & $4,32 \mathrm{a}$ & $5,15 \mathrm{~b}$ & $21,44 \mathrm{a}$ \\
$\mathrm{H}=30 \mathrm{~cm}$ e s= caixa plástica & $0,25 \mathrm{a}$ & $4,33 \mathrm{a}$ & $5,05 \mathrm{ab}$ & $21,04 \mathrm{a}$ \\
$\mathrm{H}=10 \mathrm{~cm}$ e s= metálica & $0,27 \mathrm{a}$ & $4,31 \mathrm{a}$ & $4,85 \mathrm{ab}$ & $20,67 \mathrm{a}$ \\
$\mathrm{H}=20 \mathrm{~cm}$ e s= metálica & $0,26 \mathrm{a}$ & $4,29 \mathrm{a}$ & $5,10 \mathrm{~b}$ & $21,04 \mathrm{a}$ \\
$\mathrm{H}=30 \mathrm{~cm}$ e s= metálica & $0,25 \mathrm{a}$ & $4,35 \mathrm{a}$ & $4,95 \mathrm{ab}$ & $21,19 \mathrm{a}$ \\
\hline
\end{tabular}

*Médias com mesma letra não diferem entre si, pelo teste de Tukey $(\mathrm{p}=0,05) . \mathrm{H}=$ altura de queda; s= superfície de recepção.

servada neste caso para perda de massa. Uma das possíveis explicações devese que a queda dos frutos, mesmo em baixas alturas, proporciona danos físicos que já influenciam o metabolismo do produto e conseqüentemente a perda de massa. Camargo et al. (2004), em teste realizado em laboratório, observaram uma perda de massa de $2,49 \%$ para tomates submetidos a queda de $40 \mathrm{~cm}$ em superfície metálica. Segundo Miller (2002), o dano físico provoca o rompimento da casca e este local se torna o principal responsável pela perda de água e por isso frutos com danos apresentam grandes perdas de água.

As análises químicas acidez titulável, pH e vitamina $\mathrm{C}$ não apresentaram diferença estatística ( $p>0,05)$. Na análise de teor de sólidos solúveis, a menor média foi apresentada pela testemunha e entre os outros tratamentos não houve diferença significativa. Kader et al. (1978) e MacLeod et al. (1976) também observaram que tomates submetidos a impacto apresentaram o maior valor de açúcar quando comparado à testemunha, mas estas diferenças não eram estatisticamente significativas.

Através dos resultados obtidos pelo ensaio da esfera instrumentada e com os tomates de mesa, foi observado que superfícies mais rígidas e maiores alturas de queda causam impactos de alta magnitude $(\mathrm{G}, \mathrm{m} / \mathrm{s} 2)$ e, conseqüentemente, maior incidência de danos físicos nos frutos, e o uso de superfícies protetoras de espuma reduzem consideravelmente a aceleração máxima (G) e a injúria interna nos frutos. Os resultados obtidos são indicativos que uma redução significativa na incidência em danos físicos pode ocorrer em linhas de beneficiamento e classificação através da adequação e/ou eliminação das alturas de queda conjugando com a utilização de superfícies protetoras. A etapa de embalagem em uma linha de beneficiamento, com quedas dos frutos diretamente na caixa plástica, em muitos casos superiores a $30 \mathrm{~cm}$, pode ser considerada um ponto crítico a ser modificado. Deve-se observar que esta pesquisa foi realizada somente para queda em um ponto crítico. Estudos futuros devem ser realizados para avaliar a relação entre a magnitude de impacto $(\mathrm{G}$, $\mathrm{m} / \mathrm{s} 2$ ) e a incidência de danos físicos em quedas seqüenciais semelhantes à encontradas em linhas de beneficiamento.

\section{AGRADECIMENTOS}

À FAPESP pelo financiamento do projeto UNIMAC e a CAPES pela concessão da bolsa de mestrado.

\section{REFERÊNCIAS}

BENASSI MT; ANTUNES AJ. 1988. A comparison of meta-phosphoric and oxalic acids as extractant solutions for the determination of vitamin $\mathrm{C}$ in selected vegetables. Arq. Biol. Tech 31: 507-513.
CAMARGO GGR; FERREIRA MD; ANDREUCETTI C. 2004. Testes de impacto em laboratório para tomate de mesa (Lycopersicon esculentum mill.), cultivar Débora. Horticultura Brasileira 22: 2.

CARVALHO CRL; MANTOVANIDM; CARVALHO PRN; MORAES RM. 1990. Análises Químicas de Alimentos (Manual Técnico). Campinas: Instituto de Tecnologia de Alimentos-ITAL. 121p.

CEAGESP. 2000. Classificação de Tomate. Programa Horti\&Fruti. 3p

FAO-FOOD AND AGRICULTURE ORGANIZATION OF THE UNITED NATIONS. 2005, 11 de março. Produção agrícola, 2004. Disponível em http://apps.fao.org.

HALSEY LH. 1955. Preliminary studies of bruising of 'turning' and 'pink' tomatoes caused by handling practices. Proceedings of the Florida State Horticultural Society 68: 240243.

HATTON TT; REEDER WF. 1963. Effect of field and packinghouse handling on bruising of Florida tomatoes. Proceedings of the Florida State Horticultural Society 76: 301-304.

HONÓRIO SL; MORETTI CL. 2002. Fisiologia pós-colheita de frutas e hortaliças. In: CORTEZ LAB; HONÓRIO SL; MORETTI CL. Resfriamento de frutas e hortaliças. Brasília: Embrapa Hortaliças. 428p.

HYDE GM; ZHANG W. 1992. Apple bruising research update: packingline impact evaluations. Tree Fruit Postharvest Journal 3: 12-15.

KADER AA; MORRIS LL; STEVENS MA; ALBRIGHT-HOLTON M. 1978. Composition and flavor Quality of Fresh Market Tomatoes as Influenced by some postharvest handling procedures. J. Amer. Soc. Hort. Sci 103: 6-13.

MacLEOD RF; KADER AA. 1976. MORRIS LL. Stimulation of ethylene and $\mathrm{CO} 2$ production of mature green tomatoes by impact bruising. HortScience 11: 604-606.

MILLER WM; WARDOWSKI WF; GRIERSON W. 2001. Packingline Machinery for Florida Citrus Packinghouses. Extension Bulletin 239. Florida Cooperative Extension Service. Institute of Food and Agricultural Sciences, University of Florida. 26p. Disponível em http://edis.ifas.ufl.edu/BODY_AE184.

MILLER AR. 2002. Harvest and handling injury: physiology, boichemistry, and detection. In: BARTZ JA. Postharvest physiology and pathology of vegetables: Second edition, revised and expanded. New York: Marcel Dekker Incorporated. 177p.

MORETTI CL; SARGENT SA; HUBER DJ; CALBO AG; PUSCHMANN R. 1998. Chemical composition and physical properties of pericarp, locule and placental tissues of tomatoes with internal bruising. Journal of the American Society for Horticultural Science 123: 656-660.

SARGENT SA; BRECHT JK; ZOELLNER JJ. 1992. Sensitivity of tomatoes at mature-green and breaker ripeness stages to internal bruising. Journal of the American Society for Horticultural Science 117: 119-123.

TIMM EJ; BROWN GK. 1991. Impacts recorded on avocado, papaya, and pineapple packing lines. Applied engineering in Agriculture 7: 418-422.(Footnotes)

${ }^{1}$ Este trabalho faz parte do Projeto UNIMAC-Unidade Móvel de Auxílio à Colheita, financiado pela FAPESP. 